\title{
BOLUS PROPAGATION IN PIG URETER IN VITRO
}

\author{
R. VAN MASTRIGT, PH.D. \\ E. A. TAUECCHIO \\ From the Department of Urology, Erasmus University \\ of Rotterdam, The Netherlands
}

\begin{abstract}
Pig ureters were made to propagate injected fluid boluses by electrical stimulation in vitro. The propagation velocity was determined from EMG measurements made at several points along the ureter. It was found that this velocity varied both along the ureter and as a function of time, and that it was related to the contraction pressure but not to the bolus size.
\end{abstract}

Under normal flow conditions, the ureter transports urine in isolated boluses. ${ }^{1,2}$ Each bolus of urine is propelled by a local, moving contraction of the ureteral wall. ${ }^{3 \cdot 6}$ The electrical (EMG) signal which accompanies the mechanical activity can be measured and modelled. ${ }^{7-9}$ The present study was undertaken to investigate this transport mechanism. The propagation velocity was calculated from the electrical signal measured at a number of points along the ureter, and the influence of various parameters on the velocity was studied.

\section{Material and Methods}

Experiments were performed on 8 freshly dissected ureters from pigs which were sacrificed for cardiovascular experiments. Various drugs had been administered to the pigs, including beta-blockers and calcium-antagonists, sometimes in very large doses, so that an influence on the smooth musculature of the ureter could not be excluded. The ureters were mounted horizontally in a groove in a block of plastic which was kept at $37^{\circ} \mathrm{C}$ by circulating hot water through channels bored in it.

The pyeloureteral junction was fixed between steel electrodes of approximately 3 by 5 $\mathrm{mm}$. The ureterovesical junction was tied over a Millar microtip pressure-measuring catheter. The size of the catheter was initially $8 \mathrm{~F}$, and later $4 \mathrm{~F}$. The groove was filled with a modified Krebs solution of the following composition in $\mathrm{mMol} / \mathrm{L}, \mathrm{Na}^{+} 143, \mathrm{Ca}^{2+} 1.9, \mathrm{~K}^{+} 5.9, \mathrm{Mg}^{2+}$ $1.18, \mathrm{Cl}^{-} 126.5, \mathrm{SO}_{4}{ }^{2-} 1.18, \mathrm{H}_{2} \mathrm{PO}_{4}{ }^{-} 1.2$, $\mathrm{HCO}_{3}{ }^{-} 25.01$, glucose 11 . The fluid was constantly refreshed by a shower system of 9 injection needles which continuously dripped it on to the ureter. From the groove the fluid was circulated through an external, heated container, where it was aerated with 95 per cent oxygen and 5 per cent carbon dioxide, and pumped back into the shower. During stimulation of the ureter the shower was temporarily stopped.

Four pairs of silver wires of $0.1-\mathrm{mm}$ diameter were inserted into the ureter at regularly spaced intervals and were connected to four differential EMG amplifiers set to band width of $5 \mathrm{~Hz}$ to $1 \mathrm{kHz}$ and full range of $200 \mathrm{uV}$. Boluses of the Krebs solution were injected into the ureter through a small tube inserted in the pyeloureteral junction, and the ureter was stimulated with a $10-\mathrm{V}, 100-\mathrm{Hz}$ rectangular wave for $0.5 \mathrm{~s}$. If the stimulation was successful, the injected bolus, or part of it, was transported down the ureter.

The following signals were registered on a 9channel ink jet recorder: time, stimulation 


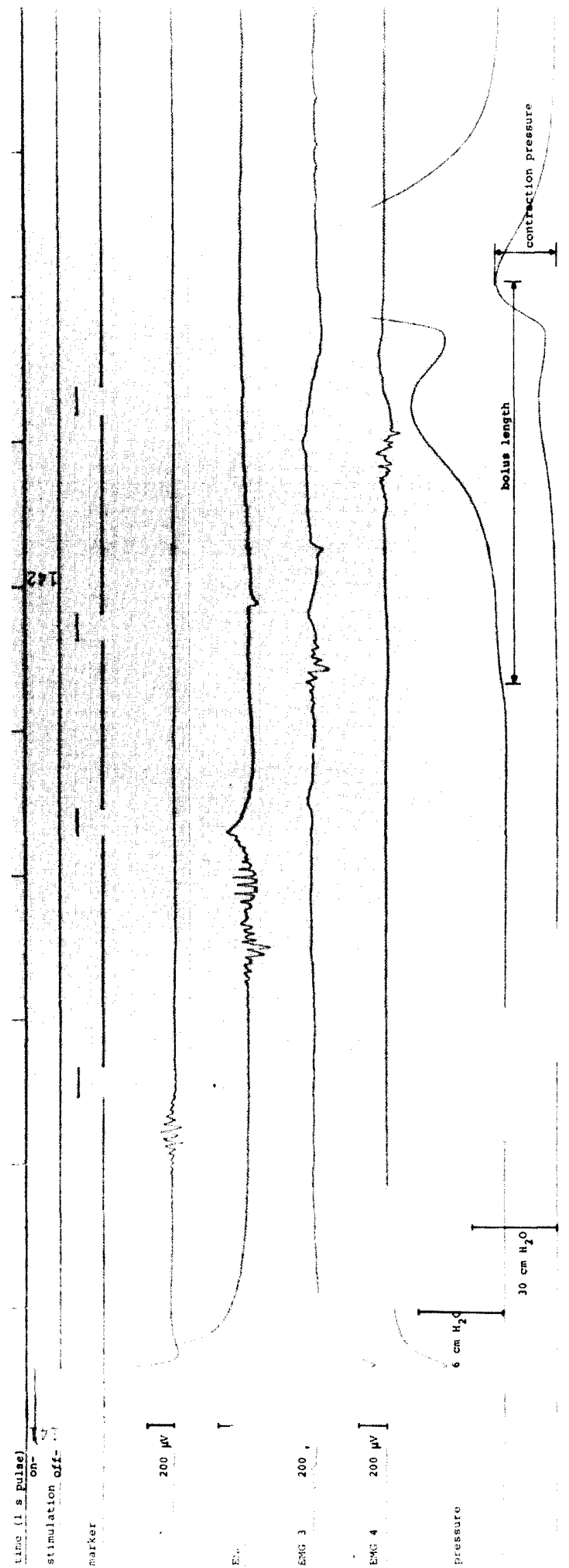

duration, marker, the four EMG channels, a the output from the pressure-measuring $c$ e ter at two different sensitivities. The tw sitivities were chosen so that both the (ve pressure in the bolus, and the (sometir pressure in the contraction ring coul ured. The marker button was $\mathrm{p}$. the ureteral contraction ring wa one of the EMG electrodes. For first 10 measurements were ta' tervals of five minutes; the ments (if possible) were tak tervals, and all subseque taken at one-minute measurements were prior injection of $\mathrm{fl}_{\mathrm{r}}$. jected volume vari increments of ' without injecte the ureter har ing measurf

The vel sive pair dividir pairs trar tir tis MG electrode pair was estimated by ing the distance between them by the time reen start of stimulation and detection of IG signal at first electrode pair. Since it is not ertain at which instant during the 0.5 -s electrical stimulation the ureteral contraction started travelling, there is an uncertainty in the calculated value of this velocity, which therefore is always presented as a minimum value.

From the recorder chart the length of the bolus was determined as the distance from the first rise of pressure to the point of maximum pressure, and the contraction pressure as the height of this maximum pressure rise (Fig. 1).

\section{Results}

In 7 of the 8 ureters, fluid boluses were propagated successfully. In several cases at the beginning of the experiment, the contractions produced by stimulation did not propagate to the end of the ureter, but by waiting for a considerable time ( 1 to 2 hours) and injecting several large boluses, proper propagation was finally established. On average about 30 measurements could be taken from 1 ureter, with a maximum of 39 measurements in 1 case. This number was dependent on the time interval 


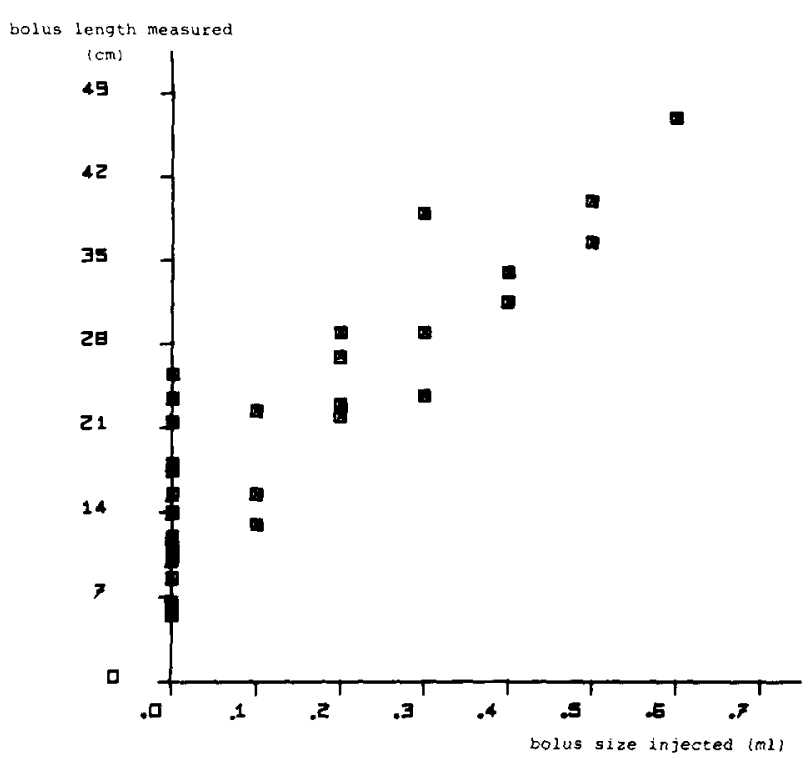

FIGURE 2. Bolus length as function of volume of injected fluid, for all measurements from one ureter. Bolus length represented as $\mathrm{cm}$ measured on chart paper is not equal to real length of bolus.

between stimulations; with the five- or twominute intervals a large number of measurements could be made, but with a one-minute interval the performance of the ureter deteriorated rapidly, soon resulting in contractions stopping before the end of the ureter. Figure 1 shows a typical result from one measurement. There is a systematic time lag of $0.2 \mathrm{~s}$ between the EMG and the marker pulse, indicating that the electrical signal travels in front of the contraction ring. The pressure signal shows a typical sequence: a bolus with a rather low pressure within it, followed by a contraction ring with a considerably higher pressure. Figure 2 shows a plot of the amount of fluid injected versus the

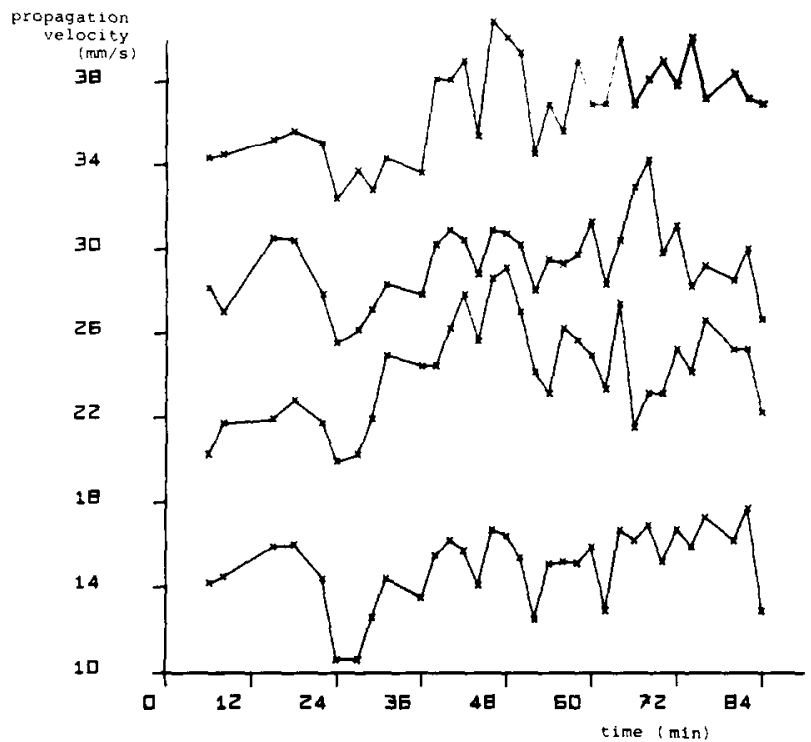

FIGURE 3. Propagation velocity of ureteral contraction between stimulation electrodes and four pairs of EMG electrodes, as function of time. Second, third, and fourth tracings are shifted, respectively, 2, 4, and 4.5 scale divisions upward with respect to first tracing. See Table I, first row, for absolute values of velocities for this ureter.

measured bolus length for this ureter. Such a plot gives an impression of the efficiency of the transport mechanism.

In this case there is a roughly linear relationship between these variables, indicating that the injected fluid is indeed transported. The relationship does not pass through the origin because for ease of measurement the bolus length was defined as the distance between the leading edge of the bolus and the maximum of the contraction pressure. It therefore includes part of the contraction ring. Even when no fluid had been injected, long boluses were occasionally transported (Fig. 2). Obviously in these cases the fluid injected previously had not been fully

TABLE I. Average propagation velocities of ureteral contraction between stimulating electrodes and four pairs of EMG electrodes, and overall average for entire ureter (seven ureters)

\begin{tabular}{clcccc}
\hline \multirow{2}{*}{ No. of } & \multicolumn{5}{c}{ Electrical Stimulation $(\mathrm{mm} / \mathrm{s})-$} \\
\cline { 2 - 6 } Measurements & \multicolumn{1}{c}{ lst* } & 1st-2nd & 2nd-3rd & 3rd-4th & Average \\
\hline $\mathrm{n}=37$ & $>15.8(4.7) \dagger$ & $16.1(2.3)$ & $13.3(1.8)$ & $19.0(3.0)$ & $16.0(2.3)$ \\
$\mathrm{n}=19$ & $>16.7(1.0)$ & $20.6(1.2)$ & $17.4(1.2)$ & $17.4(.09)$ & $18.0(.09)$ \\
$\mathrm{n}=20$ & $>17.4(2.6)$ & $19.4(2.1)$ & $14.1(0.8)$ & $17.3(.09)$ & $16.6(2.4)$ \\
$\mathrm{n}=12$ & $>25.4(2.0)$ & $22.7(1.1)$ & $26.0(3.1)$ & $34.1(2.2)$ & $25.4(4.2)$ \\
$\mathrm{n}=31$ & $>17.1(3.6)$ & $14.1(2.4)$ & $15.2(1.3)$ & $17.2(1.1)$ & $16.0(1.8)$ \\
$\mathrm{n}=39$ & $>18.6(4.5)$ & $16.2(1.1)$ & $21.1(3.1)$ & $21.5(2.8)$ & $19.3(1.5)$ \\
$\mathrm{n}=28$ & $>21.5(6.1)$ & $20.5(2.7)$ & $20.5(3.0)$ & $21.5(3.2)$ & $21.0(3.0)$ \\
\hline
\end{tabular}

${ }^{*}$ Due to uncertainty in start of the contraction during electrical stimulation, first velocity column shows a minimum value.

†Values between parentheses represent standard deviations. 

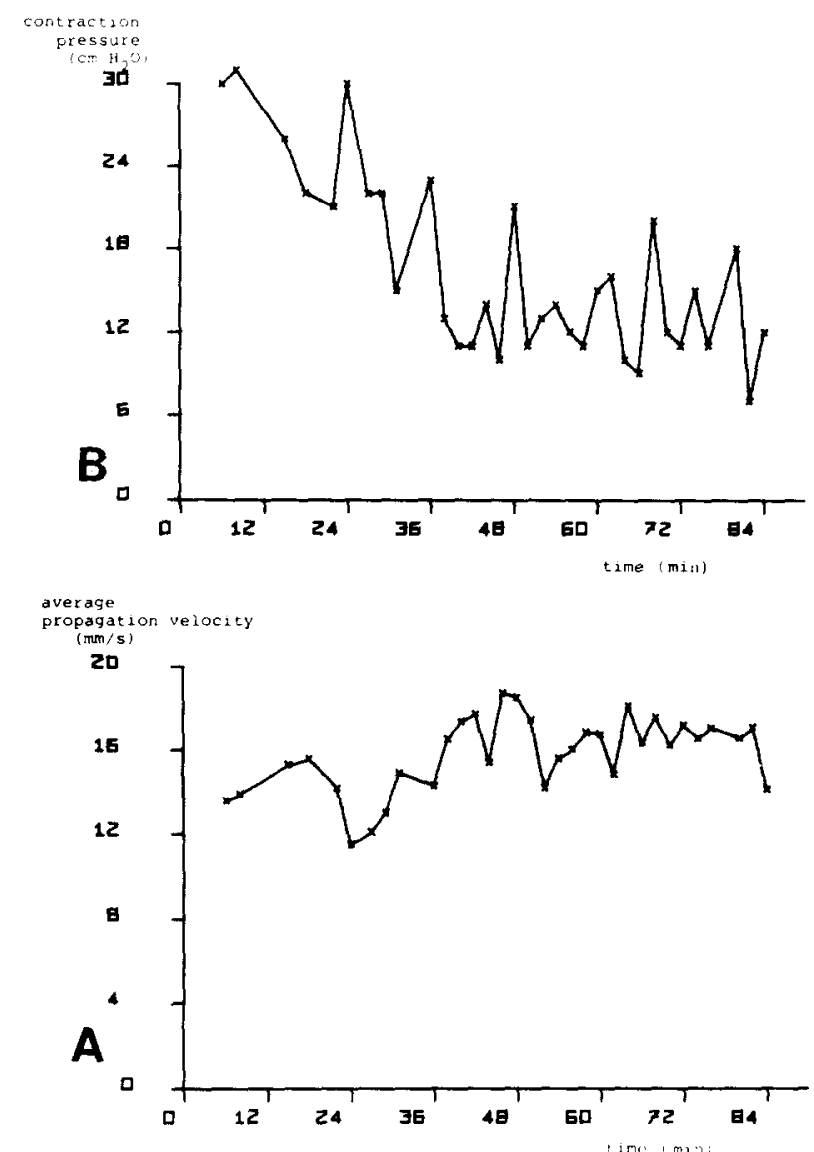

Figure 4. Average propagation velocity (calculated from data Fig. 3) as function of time (A), and associated contraction pressure as function of time (B).

ejected. In fact the highest three measurements were made very early or late in the experiment when the transport was not very efficient. In 4 of the 7 ureters, there was a linear relationship like that in Figure 2. In the other 3 ureters it was not recognizable. Figure 3 shows the 4 measured velocities as a function of time. The 4 velocities show, as well as individual random variations, a common trend. This indicates the influence of some variable which affects the propagation velocity in the entire ureter. These fluctuations are therefore also seen in the average of the 4 velocities (Fig. 4A). In 3 ureters the average velocity tended to increase as time elapsed as in Figure 4, whereas in the other 4 ureters the average velocity decreased. A systematic difference between the 4 velocities also exists (Table I). In 6 of the 7 ureters the propagation velocity in the distal section was higher than in the third section. In the last 4 ureters the velocity also was higher in the most proximal section than in the second section. Although this does not seem to be the case in the first 3 ureters, the fact that only a minimum value is given for the velocity in the proximal

section should be taken into account. Thus it is probably safe to conclude that in general the propagation velocity is higher in the proximal and distal parts of the ureter than in the middle.

In 4 ureters one or more measurements were made, at the beginning or the end of the experiment, in which the propagation of a contraction stopped about half way along the ureter. In 3 of these ureters, the contraction ran significantly more slowly than normal just before stopping. An example is shown in Figure 5, where the first two measurements of the propagation velocity between the second and third pairs of electrodes (third tracing) are significantly lower than the subsequent measurements, while the fourth electrode is not reached. In the fourth ureter this effect may have been masked by a measurement error.

Figure $4 \mathrm{~B}$ shows the contraction pressure measured in 1 ureter, as a function of time. It can be seen not only that the general trend is opposite to that of the average velocity in the same ureter (Fig. 4A) but also that a number of small excursions from the general trend are oppositely directed in the two tracings. A scatter

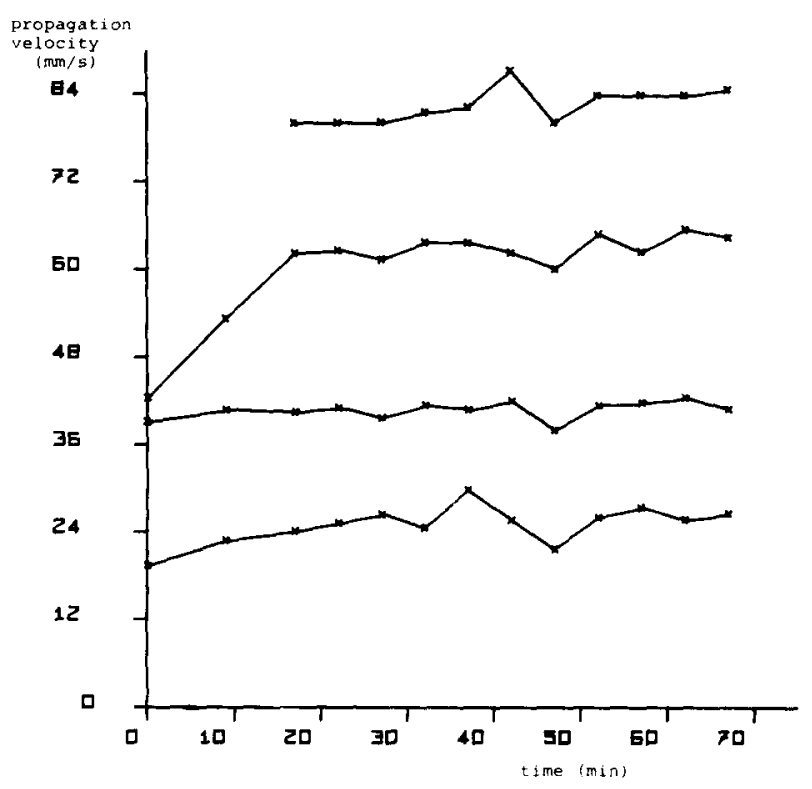

Figure 5. Propagation velocity of ureteral contraction between stimulation electrodes and four pairs of EMG electrodes, as function of time. In first two measurements ( 0 and 10 min after start of experiment) contraction stopped before reaching end of ureter. Second, third, and fourth tracings are shifted upward 1.5, 3, and 4 scale divisions, respectively, with respect to first tracing. See Table $I,(n=$ 12), for ahsolute values of velocities for this ureter. 


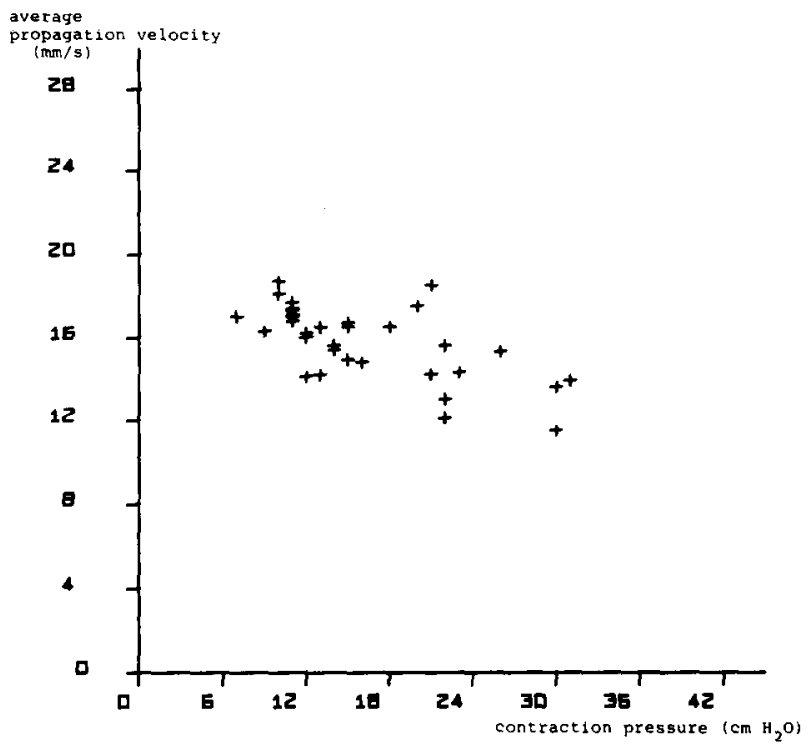

FIGURE 6. Average propagation velocity plotted as function of contraction pressure (same data as Fig. 4); Spearman's rank correlation -0.65 , significant at $1 \%$ level.

plot of the two variables demonstrates this inverse relation (Fig. 6). For these data Spearman's rank correlation coefficient is -0.65 , significant at the 1-per cent level. A similar inverse relationship was found in 3 of the 7 ureters. In the other 4 ureters the contraction pressure was either so low (3 ureters) or so high (1 ureter) that it could not be estimated reliably.

The possibility of a relationship between the propagation velocity and the length of the bolus was investigated. Scatter plots of the two variables showed no correlation in any of the ureters.

\section{Comment}

The electrical signals measured in our experiments reflect the depolarization of many cells near each pair of electrodes, so that the form of the signal cannot be accurately predicted or described. Nevertheless, there seems to be some similarity among the signals obtained from the four electrode pairs as the contraction wave passes, indicating that the form of the electrical signal reflects at least partially some unchanging characteristic of the contraction wave. The wave of pressure produced by the actual contraction travels behind the electrical signal. This time lag is probably due to the time necessary for activation of the muscle cells. ${ }^{10}$

When a bolus is propagated, the pressure within it is considerably lower than the pressure in the contraction wave. In the simplest model the pressure is uniform throughout the entire bolus. ${ }^{11}$ In our case a gradually increasing pressure often was seen (compare Fig. 1), which is probably related to a similar variation in crosssectional area of the bolus. ${ }^{12}$ However, the pressure-measuring catheter obstructs the ureter and may affect the form of the signal in our experiments. The decrease in pressure which is often observed just before the contraction ring passes may be related to this obstructive effect.

The presence of a bolus is not a necessary condition for the propagation of a contraction wave. In half of our observations the empty ureter was stimulated, and a contraction wave travelled its entire length. Furthermore, the fact that there is no relation between the velocity of propagation of the contraction wave and the size of the bolus that is propelled suggests that the straining of the ureter due to the bolus does not facilitate, nor inhibit, the contraction which follows it.

On the other hand, the propagation velocity is clearly related both to position in the ureter and to the contraction pressure, and also shows some (random?) fluctuations common to the entire ureter. The values which we found for this velocity in the pig are at the low end of the range reported for various other animals. ${ }^{25.13}$ The velocity of propagation is highest near the ends and lower in the middle of the ureter. This trend parallels that shown by the spontaneous intrinsic frequencies of ureter sections, ${ }^{9.14,15}$ although in the modelling of the electrical activity it was assumed that these latent intrinsic frequencies had no influence on the propagation velocity. ${ }^{9}$

Another interesting point concerns the influence of metabolism on the velocity. The inverse relation found between propagation velocity and contraction pressure suggests that there is a trade-off between these two variables. A similar trade-off is represented by the wellknown force-velocity relationship for contracting muscle. ${ }^{16}$ The present trade-off perhaps might be related to the force-velocity relationship, although the two velocities are, of course, different. Alternatively, it might be the result of a simpler mechanism, such as the following.

When stimulated, muscle cells have a certain probability of entering the contractile state. In a fast-moving contraction wave, the stimulation time of a given cell is shorter, resulting in a smaller cumulative chance that it contracts. This means that fewer cells contract in a faster 
contraction wave, so that the contraction pressure is lower. In contrast to this increase in velocity as time elapses, the propagation velocity decreases before a contraction stops in the middle of the ureter. Arguments based on the Wenckebach phenomenon suggest that similar behavior should be observed if contractions follow one another at (too) short a time interval. ${ }^{17}$

Summarizing, it is certainly possible to investigate bolus propagation in the ureter in vitro. The propagation velocity is not constant ${ }^{2}$ but shows considerable and understandable fluctuations depending on, among other variables, position, time, and metabolic circumstances.

P.O. Box 1738

3000 DR Rotterdam, The Netherlands

(DR, vAN MASTRIGT)

ACKNowIFDGMENT. To P. D. Verdouw for allowing us to dissect ureters from his pigs, J. J. Glerum for doing the dissections, and D. J. Griffiths for editorial assistance.

\section{References}

1. Boyarsky S, and Weinberg S: Urodynamic concepts, in Lutzeyer W, and Melchior H: Urodynamics, New York, SpringerVerlag, 1973 , p. 1.

2. Durben G, and Gerlach R: The velocity of urine bolus under different conditions, Proc First ISDU meeting, Antwerp, 1979
3. Rose JG, et al: Ureteral wall tension, Invest Urol 10: 480 (1973)

4. Weinberg SL: Ureteral function. I. Simultaneous monitoring of ureteral peristalsis, ibid 12: 103 (1974).

5. Weinberg SL, and Labay P: Ureteral function. IV. The urometrogram at increased urine output, ibid 14: 307 (1977).

6. Constantinou $\mathrm{CE}$, and Hrynczuk JR: Urodynamics of the upper urinary tract, ibid 14: 233 (1976).

7. Constantinou CE, et al: Simulated vesicoureteral reflux, Urol Int 29: 265 (1974)

8. Golenhofen K, and Hannappel J: Spontaneous generation of excitation in pyeloureteral system and the effect of adrenergic substances, in Lutzeyer $\mathrm{W}$, and Melchior $\mathrm{H}$, op cit ${ }^{1} \mathrm{p} 46$.

9. van Duyl WA, et al: Provisional model for propagation of electrical activity in upper urinary tract, Urology 12: 736 (1978).

10. van Mastrigt $R$, and Glerum JJ: Electrical stimulation of smooth muscle strips from the urinary bladder of the pig, submitted for publication.

11. Griffiths DJ, and Notschaele C: The mechanics of urine transport in the upper urinary tract. 1 . The dynamics of the isolated bolus, Neurourol Urodyn 2: 155 (1983).

12. Djurhuus J, Constantinou CE, Andersen HR, and Jorgensen F: Bolus dynamics in ureter, Proc First ISDU meeting, Antwerp, 1979.

13. Vereecken R: Dynamical aspects of urine transport in the ureter, Thesis, Catholic university Leuven (Belgium), 1973.

14. Gruber $\mathrm{CM}$ : The peristaltic and antiperistaltic movements in excised ureters as affected by drugs, J Urol 20: 27 (1928).

15. Shiratori $T$, and Kinoshita $H$ : Electromyographic studies on urinary tract. III. Influence of punching and cutting the ureters of dogs on their EMGs, Tokohu J Exp Med 73: 159 (1961).

16. van Mastrigt $R$, and van Duyl WA: Mechanics of detrusor contraction. Determination of contractility, in Coolsaet BLRA, and van Duyl WA: Principles of Bladder Function and Urodynamics, Utrecht, Lameris Instrumenten, 1981, p 31.

17. van Duyl WA, and Groot RFH: Evaluation of a model for the propagation of electrical activity along the ureter (abstr) Proc Fourth ISDU meeting, Utrecht, The Netherlands (1982). 